\title{
Complete response in a patient with gynecological hidradenocarcinoma treated with exclusive external beam radiotherapy and brachytherapy: a case report
}

\author{
Flavio Giannelli, MD', llaria Chiola, MD², Liliana Belgioia, MD1,2, Stefania Garelli, MPh³, Alice Pastorino, MD²,

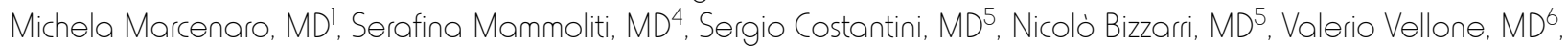 \\ Salvina Barra, MD', Renzo Corvò, MD',2 \\ 'Radiation Oncology Department, Policlinico San Martino Hospital, ${ }^{2}$ Health Science Department (DISSAL), University of Genova, \\ ${ }^{3}$ Medical Physics Department, Policlinico San Martino Hospital, ${ }^{4}$ Medical Oncology Department, Policlinico San Martino Hospital, \\ ${ }^{5}$ Gynecological Department, Policlinico San Martino Hospital, ${ }^{6}$ Pathological Anatomy Department, Policlinico San Martino Hospital, Genova, Italy
}

\begin{abstract}
Hidradenocarcinoma (HC) is a very rare disease. This case report illustrates a successful treatment of a 60-year-old woman with vulvo-vaginal localization of hidradenocarcinoma treated with external beam radiotherapy delivered by helical tomotherapy with a simultaneous integrated boost (SIB), followed by brachytherapy. External beam radiotherapy dose prescription was $50.4 \mathrm{~Gy}$ in 28 fractions, five fractions per week to whole pelvis (planning target volume 1 - PTV1), 60.2 Gy in 28 fractions to SIB1 (fundus of uterus and right inguinal node), and 58.8 Gy in 28 fractions to SIB2 (lower/middle third of vagina, paraurethral region and right inguinal lymph nodes). Brachytherapy dose prescription was 28 Gy in 4 fractions for cervix, fundus of uterus and upper third of vagina (HR-CTV1), and 22 Gy in 4 fractions to middle third of vagina and paraurethral region (HR-CTV2). $\mathrm{D}_{90}$ for whole treatment was 91.9 Gy and 86.0 Gy for HR-CTV1 and HR-CTV2, respectively. Patient remained 12-months disease-free without treatment related side effects.

J Contemp Brachytherapy 2017; 9, 6: 572-578 DOI: https://doi.org/10.5114/jcb.2017.71554
\end{abstract}

Key words: high-dose-rate (HDR) brachytherapy, image-guide radiotherapy (IGRT), vulvo-vaginal hidradenocarcinoma.

\section{Purpose}

Hidradenocarcinoma $(\mathrm{HC})$ is a rare malignant adnexal tumor, it represents approximately $6 \%$ of malignant eccrine cancers and its incidence is about $0.001 \%$ of all malignant tumors [1]. Hidradenocarcinoma affects mainly females from the fifth to the seventh decade of life [2]. The head and neck are the most common sites of HC, but occasionally it can occur in other locations [3,4]. Despite the fact that $\mathrm{HC}$ has an aggressive behavior, it may remain asymptomatic for some time. The first site of metastatic spread is lymph nodes [5]. Hidradenocarcinoma arising from the vulva is an extremely rare condition and so far, only few cases have been reported [6]. Here, we report the case of a woman with vulvar localization of $\mathrm{HC}$ who was referred to our Institution and to be considered for radical radiotherapy after previous unsuccessful chemotherapy.

\section{Case report}

In October 2015, a 60-years-old Caucasian woman, with no relevant past medical history except for removal of a benign breast nodule, was referred to our department reporting few episodes of vaginal bleeding, which started 6 months earlier. Gynecological examination revealed the presence of a circumferential vaginal mass arising from the introitus, and involving the lower and mid third of vagina. Rectal examination was suspicious for parametrial infiltration. Magnetic resonance imaging (MRI) revealed abnormal circumferential thickening of the vagina, mainly located on the anterior and left vaginal wall, with signs of infiltration of the left elevator ani-muscle and of the postero-basal bladder wall. In addition, there was a $28 \times$ $25 \mathrm{~mm}^{2}$ solid lesion in the uterine cervix, strongly suspicious for a malignancy with sign of bilateral parametrial infiltration. The lesion extended to the uterine cavity and 
a $3.5 \times 2 \mathrm{~cm}^{2}$ right lymphoadenomegaly was noted (Figure 1A-B). The subsequent 18-fluorodeoxyglucose positron emission tomography (18-FDG PET/CT) confirmed MRI findings with pathologic uptake of uterus-vagina (standardized uptake value [SUV] 7), rectum (SUV 10.3), and right inguinal nodes (SUV 6.1). A colonoscopy with biopsy was performed to evaluate if there was a rectal involvement, and it revealed an adenoma at the level of rectosigmoid junction. Tumor markers were not elevated (squamous cell carcinoma antigen: $\mathrm{SCC}=0.80$, and cancer antigen 125 [CA-125] = 23.40). Computed tomography (CT) scan of chest and abdomen showed no evidence of distant metastasis. The patient underwent incisional biopsy of the vaginal introitus. Histological examination of the surgical specimen revealed a lobulated infiltrating neoplasm (Figure 2A) constituted by different cell populations. The first constituted by columnar apocrine cells organized in irregular cystic, tubular, and papillary structures diffusely intermingled with squamous and myoepithelial cells. All the populations showed a mild degree of atypia, pleomorphism, and scattered mitotic figures. The neoplastic epithelial-stromal interface showed significant desmoplasia strongly suggesting pushing infiltration (Figure 2B). The multiphasic nature of the observed neoplasm was confirmed by immunohistochemistry testing, showing a strong positivity of citokeratin 7 in the apocrine component (Figure 2C), no staining for citokeratin 20 in all the components (Figure 2D), a strong positivity for p63 and citokeratin $34 \beta E 12$ in the squamous and myoepithelial components (Figure 2E). Interestingly, we observed a strong and diffuse positivity for p16 (Figure $2 \mathrm{~F}$ ) in the squamous component, similar to that observed in human papilloma virus (HPV)-related squamous dysplasia of cervix and vulva, while the apocrine component showed only scattered positivity. No stain for p53 was observed. On the base of morphology and immunophenotyping, and according to the clinical staging, a final diagnosis of $\mathrm{HC}$ was made.

A further biopsy at cervical level was required, but it was not feasible due to the stenosis induced by the disease. A radical surgery, as a first treatment option was considered not possible due to the disease extension, and after multidisciplinary team discussion, the patient was treated with capecitabine-based chemotherapy (CT) from

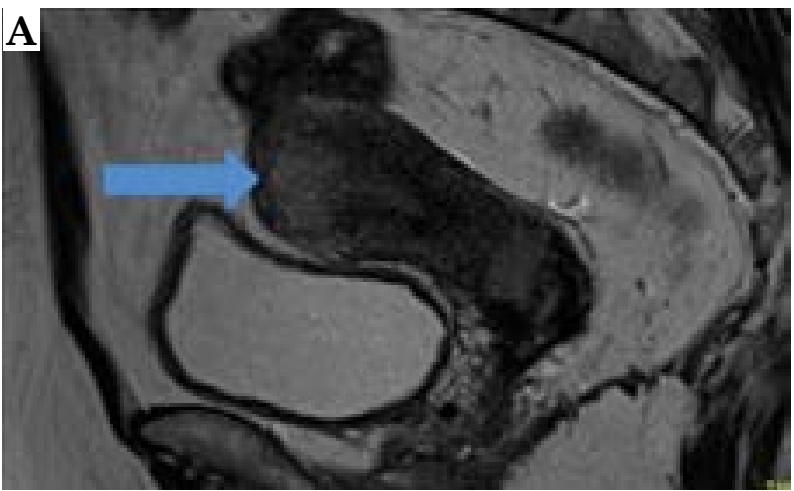

March to September 2015. After six cycles, stable disease was confirmed by clinical examination and radiological investigations. Revaluation MRI showed solid tissue to the medial rectum $10 \mathrm{~cm}$ from anal margin. 18-FDG PET/CT confirmed MRI detection, with pathologic uptake in uterus-vagina (SUV 5.8), rectum (SUV 15), and right inguinal nodes (SUV 6.9). A second colonoscopy was performed and the previously detected adenoma was endoscopically resected. The patient was considered non-respondent to chemotherapy and was referred for exclusive radiation treatment.

\section{Radiotherapy technique}

Radiotherapy treatment was divided in two phases: a first phase of external beam radiotherapy (EBRT) delivered by helical tomotherapy with simultaneous integrated boost (SIB), followed by a second brachytherapy phase. A simulation CT scan with $2.5 \mathrm{~mm}$ slices was co-registered with post chemotherapy MRI, and 18-FDG PET/CT in order to delineate the target volumes and organs at risk (OAR). The following volumes were identified: gross tumor volume (GTV), clinical target volume (CTV) whole pelvis (CTV1) including uterus, vagina, anus elevator muscle, and external and internal iliac, obturators inguinal nodes, and simultaneous integrated boosts (SIB) considering the area of metabolic uptake at 18-FDG PET/CT. SIB1 included fundus of uterus and right inguinal node, SIB2 lower/middle third of vagina, paraurethral region and right inguinal lymph nodes. The planning target volume (PTV) were defined adding $5 \mathrm{~mm}$ margin to CTV. Prescription doses were $50.4 \mathrm{~Gy}$ in 28 fractions ( 5 fractions per week) to PTV1, 60.2 Gy in 28 fractions to SIB1, and 58.8 Gy in 28 fractions to SIB2 (Table 1). This radiotherapy schedule was chosen taking into account subsequent brachytherapy treatment contribution.

Hi-Art Planning Station with Field Width $2.5 \mathrm{~cm}$ Modulation Factor 2.7 and Pitch 0.287 was used for EBRT. Duration of treatment was $8.3 \mathrm{~min}$. Before each treatment fraction, a MegaVoltage CT scan was performed for patient positioning. Image matching was done daily with an automatic bone algorithm supervised by experienced medical staff. The following goals were planned: at least $95 \%$ of the volume was covered by $95 \%$ of the dose, at

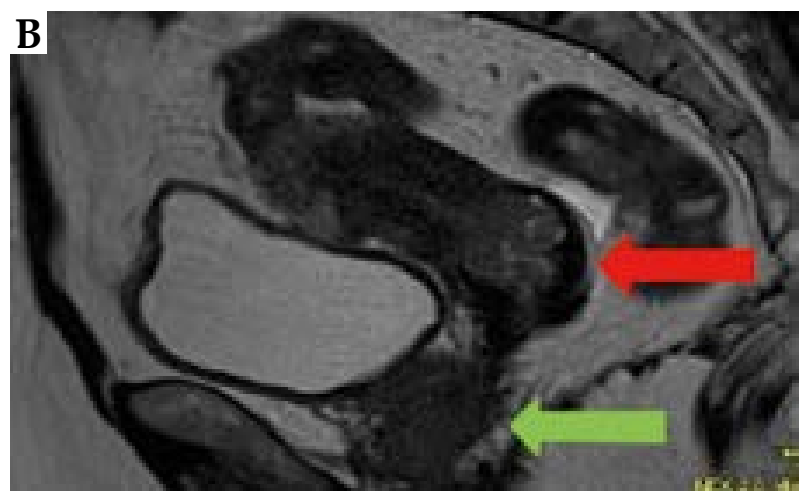

Fig. 1. Magnetic resonance imaging at diagnosis. A) Blue arrow indicates fundus uteri disease $(34 \times 20 \mathrm{~mm})$, B) red arrow indicates cervix disease $(28 \times 25 \mathrm{~mm})$, and green arrow signs of infiltration of the elevator ani-muscle and of the postero-basal bladder wall and third middle-inferior vagina and urethra 

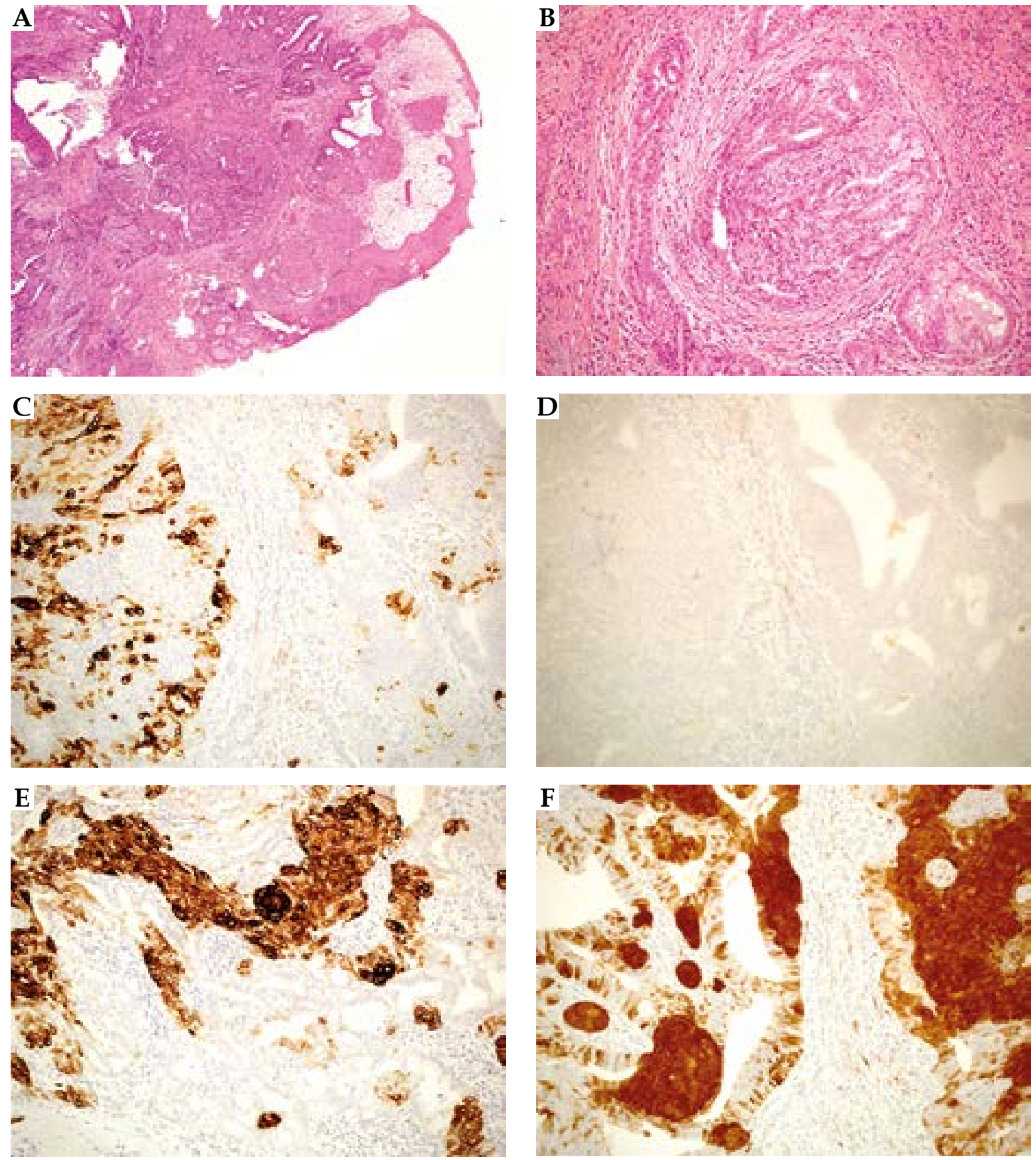

Fig. 2. Histological imaging. A) A lobulated, pushing infiltrative neoplasm, overlined by squamous epithelium (hematoxylin/ eosin; 40×). B) The neoplasm appear constituted by two, intermingled cell populations one with squamous features, the latter with apocrine feature. The epithelial-stromal junction show aspect of desmoplasia suggestive for pushing infiltration (hematoxylin/eosin; 200×). C) CK7 positivity in apocrine elements (IHC; 200×). D) No stain for CK20 (IHC; 200×). E) CK34ßE12 positivity in squamous and myoepithelial elements (IHC; 200×). F) Diffuse and intense stain for p16 in squamous and myoepithelial elements, scattered positivity in apocrine elements (IHC; 200×)

least $99 \%$ of the volume was covered by $90 \%$ of the dose for all PTVs, and maximum dose for $\mathrm{PTV}_{\mathrm{SIB} 1}$ and for $\mathrm{PTV}_{\mathrm{SIB} 2}$ was $62.25 \mathrm{~Gy}$ and $61.60 \mathrm{~Gy}$, respectively.

The main organs at risk (OAR) were: bladder, urethra, small bowel, large bowel, head and neck bilateral femo- ral, and pelvic bones. At the end of EBRT, the patient underwent clinical and radiological assessment with partial response. External beam radiotherapy was followed by 3D image-guided brachytherapy (3D IGBT) high-doserate (HDR) (MicroSelectron HDR, Elekta, Sweden). Each 
Table 1. Dose and volume description of external beam radiotherapy and brachytherapy

\begin{tabular}{|c|c|c|}
\hline & Volumes & Dose \\
\hline \multicolumn{3}{|l|}{ EBRT } \\
\hline PTV1 & Whole pelvic & $50.4 \mathrm{~Gy}$ in $28 \mathrm{fx}$ \\
\hline SIB1 & Fundus of uterus & 60.2 Gy in $28 \mathrm{fx}$ \\
\hline SIB2 & $\begin{array}{l}\text { Lower/middle third } \\
\text { of vagina paraurethral } \\
\text { region and right inguinal } \\
\text { lymph nodes }\end{array}$ & $58.8 \mathrm{~Gy}$ in $28 \mathrm{fx}$ \\
\hline \multicolumn{3}{|c|}{ Brachytherapy } \\
\hline HR-CTV1 & $\begin{array}{c}\text { Cervix, fundus of uterus, } \\
\text { upper third of vagina }\end{array}$ & 28 Gy in $4 \mathrm{fx}$ \\
\hline HR-CTV2 & $\begin{array}{l}\text { Middle third of vagina } \\
\text { and paraurethral region }\end{array}$ & 22 Gy in $4 \mathrm{fx}$ \\
\hline
\end{tabular}

EBRT - external beam radiotherapy, PTV - planning treatment volume, SIB - simultaneous integrated boost, HR-CTV - high-risk clinical target volume

brachytherapy session was performed under general anesthesia and ultrasound guidance to evaluate the appropriate positioning of the Fletcher CT/MRI applicator in the uterine cavity. After each insertion of the tandem and ovoid, the patient underwent 3T T2-weigthed MRI for the contouring of GTV, high-risk CTV (HR-CTV), and intermediate risk CTV (IR-CTV) [7]. Oncentra Masterplan TCS version 3.1.5 (Elekta, Stockholm, Sweden) was used for dose calculation with advanced collapsed cone engine (ACE) algorithm. This dose calculation algorithm

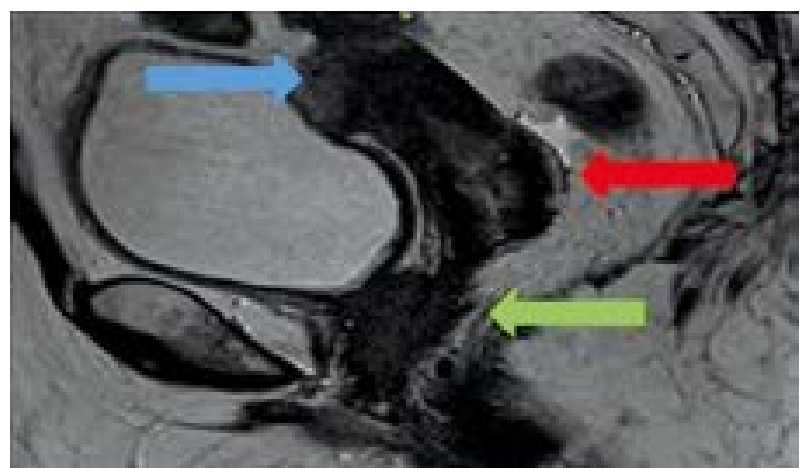

Fig. 3. Magnetic resonance imaging post-external beam radiotherapy. Blue arrow: volumetric reduction fundus uteri $(20 \times 12 \mathrm{~mm})$, red arrow: volumetric reduction of cervix disease $(20 \times 15 \mathrm{~mm})$, green arrow: stable disease of third middle-inferior vagina

for brachytherapy is based on the TG-186 recommendations, as published by the AAPM workgroup in 2012. The dose volume histograms (DVH) related to HR-CTV, IR-CTV, and organs at risk (OAR) were evaluated, taking into account in particular $\mathrm{D}_{90}$ for the CTV and $\mathrm{D}_{2 \mathrm{cc}}$ $\mathrm{D}_{1 \mathrm{cc}}, \mathrm{D}_{0,1 \mathrm{cc}}$ for OAR (rectum, sigma, bowel, bladder, and urethral). Restaging MRI following EBRT showed a moderate reduction of vaginal tissue with right paraurethral extension, and relevant reduction of cervical and fundus of uterus disease (Figure 3). Therefore, it was decided to identify two volumes called HR (high risk), which included: HR-CTV1, including cervix, fundus of uterus, and upper third of vagina, HR-CTV2 including middle third
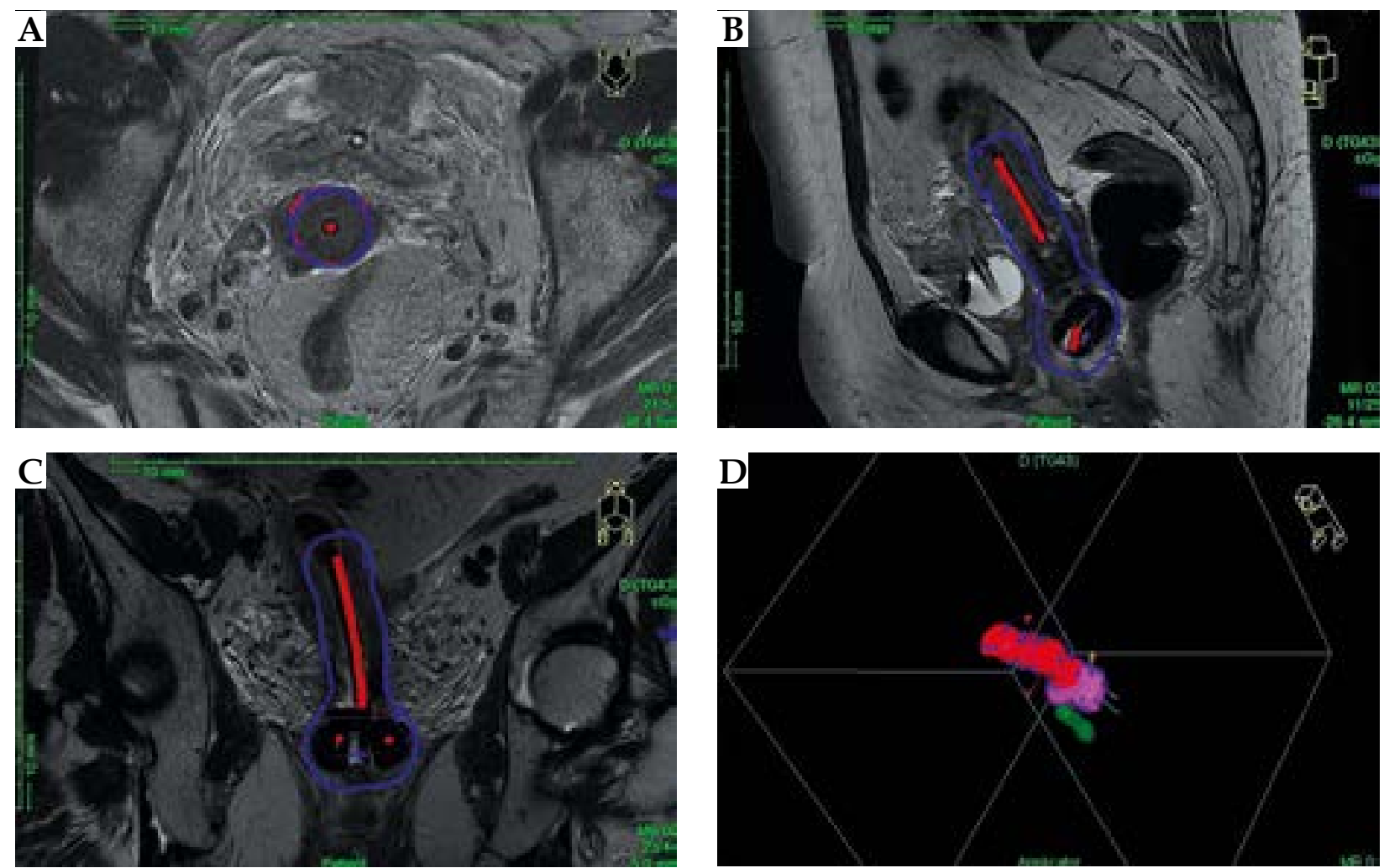

Fig. 4. Treatment planning and relative isodoses for HR-CTV1. Red line indicate HR-CTV1, blue line 7 Gy isodose 
of vagina, and paraurethral region. The doses prescribed were 28 Gy in 4 fractions to HR-CTV1 (Figure 4), 22 Gy in 4 fractions to HR-CTV2 (Figure 5). The total dose of EBRT + IGBT was calculated with a dose equivalent of 2 Gy $\left(E_{2}\right)$, assuming $\alpha / \beta=10$ for $\mathrm{T}$ and $\alpha / \beta=3$ for the OAR, with the objective of $\mathrm{D}_{90} \mathrm{HR}-\mathrm{CTV} \geq 84 \mathrm{~Gy}_{10}, \mathrm{D}_{2 \mathrm{cc}}$ $\leq 70-75 \mathrm{~Gy}_{3}, \leq 90 \mathrm{~Gy}_{3}$, and $\leq 60 \mathrm{~Gy}_{3}$ for rectum, bladder, and bowel, respectively, $D_{0,1 c c} \leq 80-85 \mathrm{~Gy}_{3}$ for urethra. $\mathrm{D}_{90}$ was $91.9 \mathrm{~Gy}$ in the HR-CTV1 and 86.0 Gy in the HR-CTV2, $\mathrm{D}_{2 \mathrm{cc}}=67.4 \mathrm{~Gy}, \mathrm{D}_{1 \mathrm{cc}}=71.2 \mathrm{~Gy}, \mathrm{D}_{0,1 \mathrm{cc}}=79.3 \mathrm{~Gy}$ for rectum, $\mathrm{D}_{2 \mathrm{cc}}=75.5 \mathrm{~Gy}, \mathrm{D}_{1 \mathrm{cc}}=80 \mathrm{~Gy}, \mathrm{D}_{0,1 \mathrm{cc}}=91.1 \mathrm{~Gy}$ for bladder, $\mathrm{D}_{2 \mathrm{cc}}=59.8 \mathrm{~Gy}, \mathrm{D}_{1 \mathrm{cc}}=62.8 \mathrm{~Gy}, \mathrm{D}_{0,1 \mathrm{cc}}=69.5 \mathrm{~Gy}$ for sigmoid, $\mathrm{D}_{2 \mathrm{cc}}=59.8 \mathrm{~Gy}, \mathrm{D}_{1 \mathrm{cc}}=62.8 \mathrm{~Gy}, \mathrm{D}_{0,1 \mathrm{cc}}=69.5 \mathrm{~Gy}$ for bowels, and $\mathrm{D}_{0,1 \mathrm{cc}}=75.4 \mathrm{~Gy}$ for urethra. Patient reported a grade 2 radiation-correlated cystitis (according to CTCAE v. 4.0) and an episode of urinary retention grade 2 (CTCAE $\mathrm{v}$. 4.0), which resolved completely with clinical treatment [8].

\section{Follow-up}

The first radiological follow-up was performed 3 months after the end of treatment with a gynecological examination, MRI, and CT scans, and tumor markers evaluation with significant dimensional reduction but persistent tumor (Figure 6). One month later the subsequent PET/CT showed a complete metabolic response, and the subsequent MRI performed at 6 months from the end of therapy confirmed this datum (Figure 7). At date, there were no relevant late side effects. The patient did not present any recurrence until February 2017. One year after the end of radiotherapy, a CT scan revealed
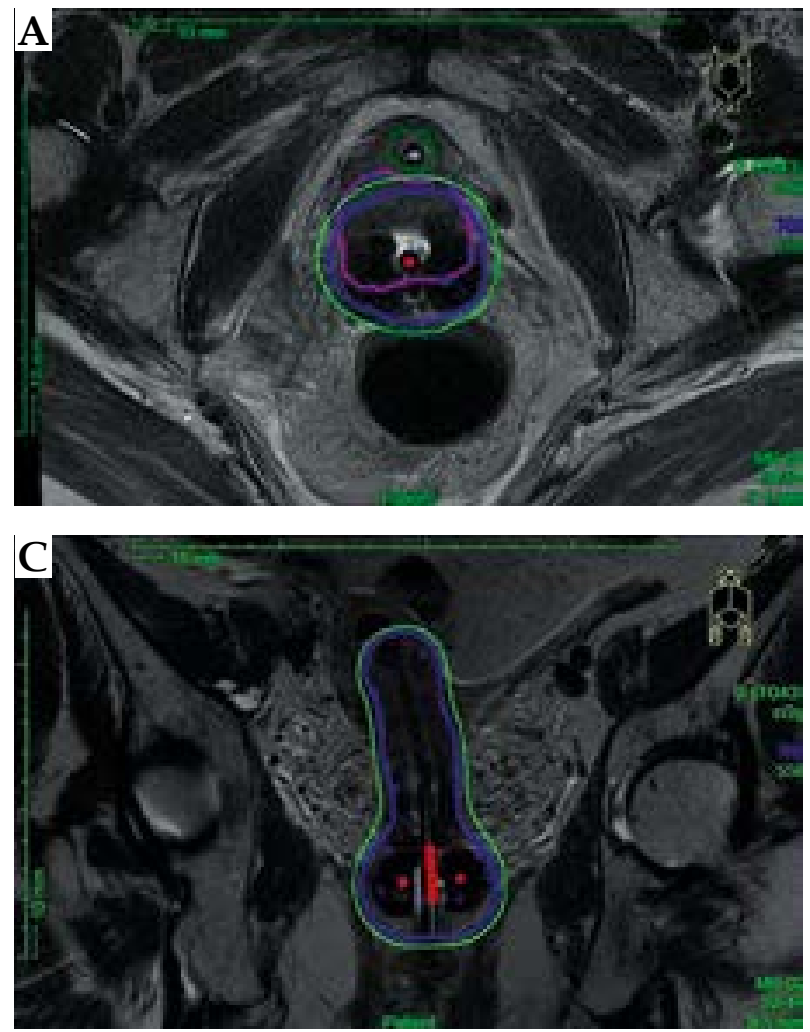

a right lung nodule with avid 18-FDG uptake at PET/CT. The patient underwent stereotactic body irradiation (SBRT) (48 Gy in 4 fractions). The subsequent PET/CT performed at 3 months showed a reduced 18-FDG uptake of the treated nodule and the appearance of a contralateral lung nodule. At date, the patient was planned for another SBRT, and then she will be re-evaluated by medical oncologist for systemic therapy.

\section{Discussion}

Hidradenocarcinoma of the vulva is a rare tumor with aggressive development and high-risk of distant metastases with five-year post-surgical survival rate smaller than $30 \%$ [9]. It arises from ano-genital mammary glandlike tissue, mainly de novo, and in rare cases, it may stem from a pre-existing hidradenoma [9]. The main location is the head and neck region, rarely on the trunk, abdomen, fingers, or scalp. Our patient presented a vulvar origin, which is exceptionally rare site; a review of the current literature reveals only 7 cases of $\mathrm{HC}$ arising from a vulva site. The diagnosis is based on histological and immunohistochemical analysis to differentiated HC from several benign (like hemangiomas, lipomas) and malignant tumors [5]. As HC is a rare disease, to date, there is no clear consensus about its treatment. However, surgery remains the cornerstone of treatment and negative margins of resection are extremely important with some data suggesting that at least a $3 \mathrm{~cm}$ margin is required $[10,11]$. Obviously, surgery depends on the stage of the tumor. In our case, the disease was locally advanced with posi-
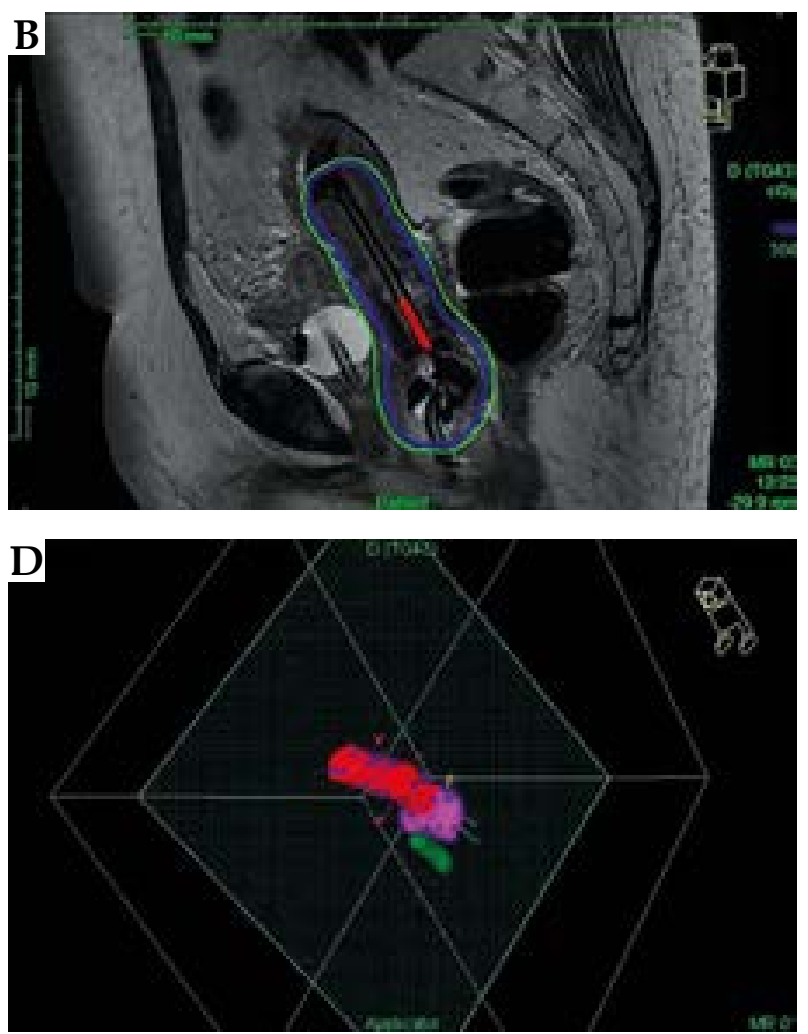

Fig. 5. Treatment planning and relative isodoses for HR-CTV2. Pink line indicate HR-CTV2, blue line 7 Gy isodose, and green line $5.5 \mathrm{~Gy}$ 


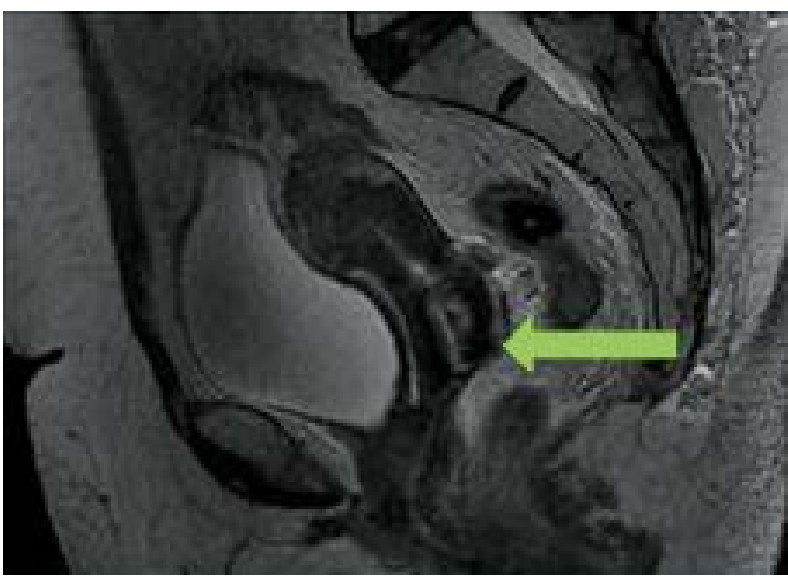

Fig. 6. Magnetic resonance imaging 3 months after external beam radiotherapy and brachytherapy. Complete response disease of cervix and fundus uteri, green arrow: partial response of third middle-inferior vagina disease

tive inguinal node and adjacent organs involvement, thus these features did not allow radical surgery even with an extremely radical operation. We started chemotherapy as a treatment to our patients with neoadjuvant intent, but with no benefit. Data regarding chemotherapy in HC are not clear and benefits have not been demonstrated, however normally, the first line treatment is based on 5-fluorouracil and capecitabine, as the molecular pattern of $\mathrm{HC}$ is similar to colon and breast cancers $[4,12,13]$. Radiotherapy is not the first choice of treatment, usually being proposed when surgery is not indicated [12,14]. Radiotherapy has been used in several cases of $\mathrm{HC}$ with conflicting results. If the aim of radiation therapy is to achieve a complete response, it is important to weight the balance of administering the highest dose possible with an acceptable morbidity; for this reason, some data recommend doses between $50-70$ Gy $[12,14]$. As our patient was deemed not operable and demonstrated poor response to chemotherapy, we decided to approach her with a high-dose radiation treatment with intensity modulated-RT and subsequent brachytherapy.

Brachytherapy is an optimal treatment option for primary and recurrent carcinomas of the vagina and of vulva; numerous brachytherapy techniques have been developed (as single channel cylinder, interstitial brachytherapy or multichannel cylinder), the decision of which brachytherapy technique to employ is influenced by multiple factors (tumor location, extent of disease, response to EBRT).

In our case, probably a better coverage of paraurethral disease could be obtained with interstitial brachytherapy, but in our institute we did not implement this technique, and the patient refused to move far away from her residence $[15,16]$.

Our patient underwent a tailored treatment that allowed us to obtain a complete response, although the wide extension of the disease at diagnosis, with acceptable short term toxicity and, at date, also long term toxicity. Despite this excellent outcome, $\mathrm{HC}$ remains an aggressive disease with poor prognosis (our patient developed lung metastasis).

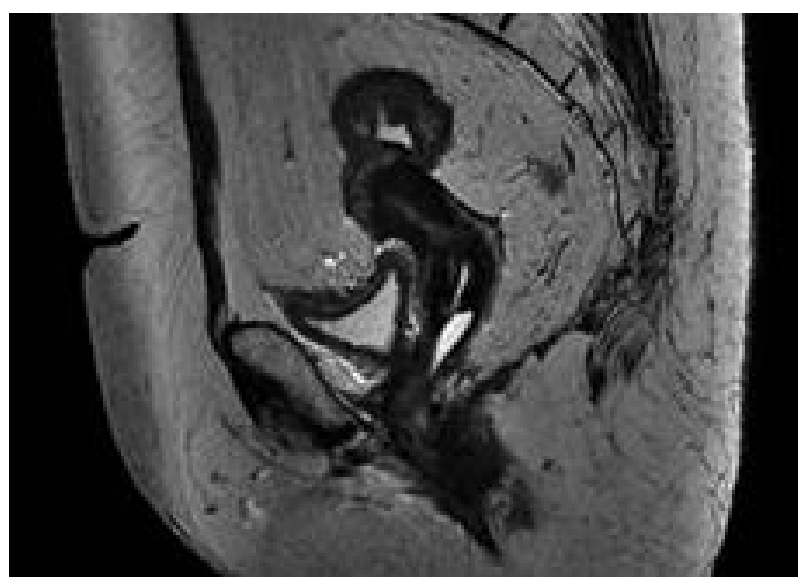

Fig. 7. Magnetic resonance imaging 6 months after external beam radiotherapy and brachytherapy. Complete response

\section{Conclusions}

The present case report shows that EBRT and brachytherapy are feasible options in the treatment of $\mathrm{HC}$, with possibility to obtain local control and acceptable tolerance. Despite this, a longer follow-up is necessary to evaluate the risk of late toxicity that could appear also after a long time from RT.

\section{Disclosure}

Authors report no conflict of interest.

\section{References}

1. Cruz DJ. Sweat gland carcinomas: a comprehensive review. Semin Diagn Pathol 1987; 4: 38-74.

2. Mehregan AH, Hashimoto K, Rahbari H. Eccrine adenocarcinoma. A clinicopathologic study of 35 cases. Arch Dermatol 1983; 119: 104-114.

3. Yavel R, Hinshaw M, Rao V et al. Hidradenomas and a hidradenocarcinoma of the scalp managed using Mohs micrographic surgery and a multidisciplinary approach: case reports and review of the literature. Dermatol Surg 2009; 35: 273-281.

4. Amel T, Olfa G, Faten H et al. Metastatic hidradenocarcinoma: Surgery and chemotherapy. N Am J Med Sci 2009; 1: 372-374.

5. Gauerke S, Driscoll JJ. Hidradenocarcinomas: a brief review and future directions. Arch Pathol Lab Med 2010; 134: 781-785.

6. Kazakov DV, Spagnolo DV, Kacerovska D et al. Lesions of anogenital mammary-like glands: an update. Adv Anat Pathol 2011; 18: 1-28.

7. Pötter R, Haie-Meder C, Van Limbergen E et al. GEC ESTRO Working Group Recommendations from gynaecological (GYN) GEC ESTRO working group (II): concepts and terms in 3D image-based treatment planning in cervix cancer brachytherapy-3D dose volume parameters and aspects of 3D image-based anatomy, radiation physics, radiobiology. Radiother Oncol 2006; 78: 67-77.

8. Common Terminology Criteria for Adverse Events (CTCAE). Version 4.0. Published: May 28, 2009 (v.4.03: June 14, 2010). U.S. Department of Health and Human Services. National Institutes of Health, National Cancer Institute.

9. Mirza I, Kloss R, Sieber SC. Malignant eccrine spiradenoma. Arch Pathol Lab Med 2002; 126: 591-594. 
10. Obaidat NA, Alsaad KO, Ghazarian D. Skin adnexal neoplasms - part 2: an approach to tumours of cutaneous sweat glands. J Clin Pathol 2007; 60: 145-159.

11. Lalya I, Hadadi K, Tazi El M et al. Radiotherapy on hidradenocarcinoma. N Am J Med Sci 2011; 3: 43-45.

12. Guillot B. From unusual cutaneous malignancies: cutaneous adnexal tumors. In: Management of rare adult tumors. Belkacemi Y, Mirimanoff R, Ozsahin M (eds.). Springer-Verlag, Paris 2009; 471-477.

13. Jouary T, Kaiafa A, Lipinski P et al. Metastatic hidradenocarcinoma: efficacy of capecitabine. Arch Dermatol 2006; 142: 1366-1367.

14. Harari PM, Shimm DS, Bangert JL et al. The role of radiotherapy in the treatment of malignant sweat gland neoplasms. Cancer 1990; 65: 1737-1740.

15. Glaser SM, Beriwal S. Brachytherapy for malignancies of the vagina in the 3D era. J Contemp Brachytherapy 2015; 7:312-318.

16. Kellas-Ślęczka S, Białas B, Fijałkowski $M$ et al. Interstitial high-dose-rate brachytherapy in locally advanced and recurrent vulvar cancer. J Contemp Brachytherapy 2016; 8: 32-40. 